\title{
Some general notes
}

\section{Translations}

All translations from the original languages, unless taken from a work already translated into English, are mine. Any mistakes or misunderstandings are therefore mine.

\section{Place Names}

In the multilingual Habsburg Empire, the language used for place names formed a source of much conflict. In this work, where a commonly accepted English version of a place name is available, it is used. Since the vast majority of references to other place names that came from the archives were in German, the German name is generally used in these instances. Where necessary, an indication is given of the name of a place as it would appear in current English usage. 